\title{
High prevalence of urinary schistosomiasis in two communities in South Darfur: implication for interventions
}

\author{
Kebede Deribe ${ }^{1 *}$, Abdeljbar Eldaw², Samir Hadziabduli, Emmanuel Kailie ${ }^{1}$, Mohamed D Omer $^{3}$, \\ Alam E Mohammed ${ }^{3}$, Tanole Jamshed ${ }^{4}$, Elmonshawe A Mohammed ${ }^{4}$, Ali Mergani ${ }^{5}$, Gafar A Ali ${ }^{6}$, \\ Khalid Babikir ${ }^{6}$, Abdulrahman Adem ${ }^{6}$, Farouq Hashim ${ }^{1}$
}

\begin{abstract}
Background: There are few data on the prevalence of schistosomiasis in Darfur. We conducted this study in response to reports of 15 laboratory confirmed cases of schistosomiasis and visible haematuria among children from two communities in South Darfur. The aim of the study was to estimate the prevalence of schistosomiasis in the area and to decide on modalities of intervention.

Methods: A cross-sectional survey involving 811 children and adults from schools and health facilities was conducted in two communities of South Darfur in March 2010. Urine samples were collected and examined for ova of Schistosoma haematobium using a sedimentation technique. A semi-structured format was used to collect socio-demographic characteristics of the participants.

Results: Eight hundred eleven (811) urine samples were collected, 415 from Alsafia and 396 from Abuselala. Of the collected samples in 56.0\% (95\% Confidence Interval (Cl); 52.6-59.4) Schistosoma eggs were found. The prevalence was high in both Abuselala $73.3 \%$ (95\% Cl; 68.9-77.6) and Alsafia 39.5\% (95\% Cl; 34.8-44.2). More males (61.7\%, 95\% $\mathrm{Cl}$; 56.5-64.9) were infected than females $(52.1 \%, 95 \% \mathrm{Cl}$; 48.2-56.0). Children in the age group 10-14 has the highest (73.0\%, 95\% Cl; 68.7-77.2) infection rate. School age children (6-15 years) are more likely to be infected than those $>15$ years (Adjusted Odds Ratio $(A O R)=2.70,95 \%$ Cl; 1.80-4.06). Individuals in Abuselala are more likely to be infected than those who live in Alsafia (AOR $=4.3,95 \% \mathrm{Cl} ; 3.2-5.9$ ).

Conclusion: The findings of this study indicate that $S$. hematobium is endemic in Alsafia and Abuselala South Darfur in Sudan with a high prevalence of infection among older children. This signifies the importance of urgent intervention through Mass Drug Administration (MDA) to halt the infection cycle and tailored health messages to targeted groups. Based on the findings MDA was conducted in the villages.
\end{abstract}

\section{Introduction}

Schistosomiasis or bilharzia is a tropical parasitic disease caused by blood-dwelling flukes of the genus Schistosoma [1]. It affects about 200 million people worldwide, and more than 650 million people live in endemic areas $[1,2]$. Urinary schistosomiasis is caused by Schistosoma haematobium and is endemic in sub-Saharan Africa, including Sudan. Estimates for this region of the world

\footnotetext{
* Correspondence: kebededeka@yahoo.com

${ }^{1}$ American Refugee Committee International, Nyala, South Darfur, Sudan

Full list of author information is available at the end of the article
}

suggest that 436 million people live at risk of infection with Schistosoma haematobium [3,4].

Although schistosomiasis is highly prevalent, the associated morbidity is low and variable [1]. However, the Global Burden of Disease Study, attributes a disability weight of 0.06 and an annual mortality of 14000 deaths per year to schistosomiasis. Based on 200 million infected people worldwide, the total number of Disability Adjusted Life Years (DALY) lost to schistosomiasis is estimated at 1.532 million per year, of which $77 \%$ are in sub-Saharan Africa [5]. New meta-analyses indicated a

\section{C) Biomed Central}


mortality estimate up to 280,000 deaths annually in subSaharan Africa alone $[4,6]$.

Although the intimate connection between conflict and Neglected Tropical Disease (NTD) such as schistosomiasis is known [7], the prevention and control of such disease in conflict and emergency settings are often neglected. In such contexts, more emphasis is given for lifesaving services and diseases of epidemic importance. If the prevention and control of such diseases do occur, they are often uncoordinated and driven by the occurrence of a cluster of cases.

American Refugee Committee International (ARC) has been working in Darfur since October 2004. This study was conducted in response to American Refugee Committee's (ARC) report of cases of schistosomiasis in Abuselal and Alsafia to State Ministry of Health (SMoH). Fifteen laboratory confirmed cases of schistosomiasis from Alsafia and visible haematuria among children from Abuselala were reported through ARC supported health facilities. Therefore this study was conducted to estimate the prevalence of schistosomiasis in the area and to decide on modalities of intervention.

\section{Materials and methods Study site}

The survey was conducted in two villages in South Darfur State, south western region of North Sudan during March 2010. The villages (Alsafia and Abuselala) are located 75 and 103 Kilometers Southwest of Nyala the capital of the state. The villages lies between Latitude N: 111252.95 and Longitiude: E: 2442 47.29. The villages have an estimated population of 5,000 (Abuselala) and 9,000 (Alsafia). The State experiences a single rainy season, typically between June and October. The villages have seasonal ponds, the inhabitants utilize the ponds for fishing, domestic purposes, and children and young adolescents swim regularly in the ponds [8]. Both areas have no history of treatment for schistosomiasis and this study is the first of its kind in this setting.

\section{Sample population and selection}

For schistosomiasis control, WHO recommends that 200-250 school-aged children sampled in each ecological zone [9]. Children in school, Khalwas (religious schools), and children and adults in health facilities were included in the study. The data was collected from 811(415 Alsafia and 396 Abuselala) individuals. A total of 323 and 329 school-age chidren were enrolled in the study from Abuselala and Alsafia respectively, which is more than the WHO minumum recomendation. In addition adults and under-five children were enrolled to see the pattern of infection acrosse age groups.

\section{Data collection}

In brief, for all individuals, age, sex and location were captured through a semi-structured format. Mass urine investigation of schistosome eggs identification was conducted. Parasitological examination of urine samples was conducted in the field by a team of trained laboratory technicians. From each subject, a $10 \mathrm{ml}$ sample of terminally voided urine was collected in a properly labeled clean and sterile specimen container between 10.00 and $14.00 / \mathrm{h}$. Centrifugation and sedimentation techniques [10] were employed to analyze the samples. $10 \mathrm{ml}$ urine was taken from the deposit of each specimen bottle after allowing to sediment for about an hour and centrifuged for 2 mins at $2000 \mathrm{rpm}$. The deposit was thereafter examined microscopically using $\times 10$ and $\times 40$ objectives for the characteristics schistosome egg. Urine samples containing egg(s) of S. haematobium were recorded as positive, while absence of eggs of schistosomes was considered as negative [10].

\section{Data management and statistical methods}

Data were edited, cleaned, and analyzed using SPSS for Windows version 16.0 (SPSS Inc., Chicago, IL, USA). To calculate the prevalence, descriptive analysis was done. Bivariate and multivariate logistic regression analysis was done to examine the association between sociodemographic variables and schistosomiasis. A simple regression model was produced to identify factors associated with infection by adjusting for age, sex and residence.

\section{Ethical considerations}

The study was approved by $\mathrm{SMoH}$, followed by the Local Health Authority. The study was explained to each participant. Individuals or caregivers were asked to consent verbally to participate in the study; only those who provided consent were registered and requested to provide samples. To document verbal consent, the name of each individual who provided verbal consent was recorded, along with the test results for their samples. Individuals who tested positive for schistosomiasis infection were treated with praziquantel, according to WHO guidelines. Due to shortage of medicine some (52) of the participants were referred to health facilities. Parents/guardians provided consent on behalf of all child participants.

\section{Results}

\section{Socio-demographic characteristics}

In this study 811 individuals were involved, 415 from Alsafia and 396 from Abuselala. Most (84.5\%) of the participants were in primary school age group. The median age was 10 (inter-quartile range (IQR): 8-13 years). 
The majority $(54.1 \%)$ of the participants were male; the socio-demographic characteristic of the participants is shown in Table 1.

\section{Prevalence of urinary schistosomiasis}

Urine samples were taken from different sample groups; students from Abuselala School, patients and clients in Abuselala PHC and in and out of school children and adults in Alsafia. In total, 454/811 (56.0\%, 95\% Confidence Interval (CI); 52.6-59.4) individuals harbored Schistosoma eggs. The overall prevalence among school age (6-15 years) children is $60.6 \%$ (76.8\% in Abuselala and $44.7 \%$ in Alsafia). The prevalence was significantly high $(73.3 \%, 95 \% \mathrm{CI}$; 68.9 77.6]) in Abuselala compared to Alsafia (39.5\%, 95\% CI; $34.8-44.2)$ ( $p<0.001)$. Similarly the prevalence was significantly high $(60.6 \%)$ among children in 6-15 years age, followed by $>15$ years (38.2\%) and $\leq 5$ children $(26.7 \%)$ ( $p<$ $0.001)$. Likewise the prevalence was significantly high (61.7\%, 95CI; 56.5-64.9) among male compared with female $(52.1 \%, 95 \% \mathrm{CI} ; 48.2-56.0)(\mathrm{p}=0.015)$. In a separate analysis, children in age groups $10-14$ and 15-19 were more likely to be infected than children in the age group $5-9$ with odds of $(\mathrm{OR}=2.90,95 \% \mathrm{CI} ; 2.08-4.06)$ and $(\mathrm{OR}=$ $2.42,95 \% \mathrm{CI}, 1.37-4.28)$ respectively.

\section{Factors associated with presence of Schistosoma eggs}

All the factors (age, sex and address) associated with presence of Schistosoma eggs were entered into logistic regression model and only age and address were independently associated. Children in the age range of 6-15 are more likely to be infected than those $>15$ years old $(\mathrm{OR}=2.70,95 \% \mathrm{CI} ; 1.80-4.06)$. Likewise those individuals who live in Abuselala were more likely to be infected than those who live in Alsafia $(\mathrm{OR}=4.31,95 \%$ CI, 3.17-5.86). (Table 2)

\section{Discussion}

The results of this study show a prevalence rate of $56 \%$ of urinary schistosomiasis among the respondents in Darfu. The result is higher than reported in previous studies in the state $[11,12]$.

Table 1 Sociodemographic characteristics of study participants South Darfur, March 2010

\begin{tabular}{llll}
\hline Variable & Category & Number & Percent \\
\hline Age in years & $\leq 5$ & 15 & 1.8 \\
& $6-15$ & 652 & 80.4 \\
& $>15$ & 144 & 17.8 \\
Address & Abuselala PHC & 193 & 23.8 \\
& Abuselala School & 203 & 25.0 \\
Sex & Alsafia all & 415 & 51.2 \\
& Male & 445 & 54.9 \\
& Female & 366 & 45.1 \\
\hline
\end{tabular}

S. haematobium has previously been reported in Southern Darfur in earlier years [13]. The snail intermediate host Bulinus truncatus is generally found in Southern Darfur where it is well adapted to pools and slow-flowing waters [14].

In Sudan the risk for schistosomiasis is widespread, especially in the major irrigation systems in the Gezira area between the Blue and White Nile Rivers - high prevalence of $S$. mansoni infection in some areas in the West Equatoria region and both $S$. mansoni and S. hematobium are highly endemic in the Upper Nile region. In the country 5 million people are infected by schistosomiasis $[15,16]$.

The higher prevalence rate of urinary schistosomiasis in this study reflects the higher level of exposure and dependence of the inhabitants on the infected water bodies. There are small ponds in the areas which are used for fishing, household utilization and swimming. Similar to other studies $[17,18]$ the prevalence of schistosomiasis is high among children in age group 10-14. The disparity in the pattern of the infection among the individuals of different age groups with school-age children having the highest infection rate and the least inflections occurring among children in pre-school age could be due to low exposure. Among the children, the females had relatively lower infection than their male counterparts. This could be due to difference in exposure status. In a separate analysis there was no significant difference in the rate of infection observed among adult females and males. Respondents in Abuselala have higher infection rate than those found in Alsafia and this could be attributed to types of water bodies and water contact practices which need further investigation.

This study has a number of limitations; therefore the findings should be interpreted in the light of these limitations. First of all, we have not collected data on environmental and lifestyles of the participant which might have implication on interpretation of the data and implication for intervention. Secondly, we did not measure intensity of the infection. Thirdly, we have included individuals attending health facility which might overestimate the prevalence of the infection. Finally we used a sedimentation method which, although recommended by WHO is less sensitive and might underestimate the prevalence.

In light of the above limitation the findings of this study suggest that $S$. hematobium is endemic in Alsafia and Abuselala South Darfur in Sudan with a high prevalence of infection among older children and affecting more male than female. In complex emergencies, health services provision usually focuses on epidemic important diseases and life saving services but often ignores endemic diseases. Although in the initial phases of an emergency, interventions should focus on the most critical 
Table 2 Prevalence of Schistosomiasis among study participants South Darfur, March 2010

\begin{tabular}{|c|c|c|c|c|c|}
\hline \multirow[t]{2}{*}{ Variables } & \multicolumn{2}{|c|}{ Test Result } & \multirow[t]{2}{*}{ COR $(95 \% \mathrm{Cl})$} & \multirow[t]{2}{*}{ AOR $(95 \% \mathrm{Cl})$} & \multirow[t]{2}{*}{ P-value } \\
\hline & Negative & Positive & & & \\
\hline Age in years & & & & & $<0.001$ \\
\hline$\leq 5$ & $11(73.3 \%)$ & $4(26.7 \%)$ & $0.59(0.18-1.94)$ & $0.69(0.20-2.43)$ & \\
\hline $6-15$ & 257 (39.4\%) & $395(60.6 \%)$ & $2.49(1.72-3.60)$ & $2.70(1.80-4.06)$ & \\
\hline$>15$ & 89 (61.8\%) & 55 (38.2\%) & 1.0 & 1.0 & \\
\hline Address & & & & & $<0.001$ \\
\hline Alsafia & $251(60.5 \%)$ & 164 (39.5\%) & 1.0 & 1.0 & \\
\hline Abuselala & $106(26.8 \%)$ & $290(73.2 \%)$ & $4.18(3.11-5.64)$ & $4.31(3.17-5.86)$ & \\
\hline Sex & & & & & $>0.05$ \\
\hline Male & 144 (39.3\%) & $222(60.7 \%)$ & $1.42(1.01-1.87)$ & $1.02(0.74-1.39)$ & \\
\hline Female & $213(47.9 \%)$ & $232(52.1 \%)$ & 1.0 & 1.0 & \\
\hline
\end{tabular}

*Adjusted for age, sex and address

diseases. In the later stages, integrated approaches towards treating tropical disease and local endemic disease should be considered.

\section{Implication for intervention}

The data on the prevalence of urinary schistosomiasis, where $56 \%$ of the volunteers examined harbored S. haematobium ova in their urine, indicated endemicity of the infection. World Health Organization categorizes level of infection based on prevalence among school age children. The guideline recommends that, in communities with schistosomiasis, and a prevalence of $10 \%$ up to $50 \%$, school-aged children and high-risk groups of adults should be treated with praziquantel once every two years. In communities where prevalence is $50 \%$ and above, the same groups should be treated once a year [2]. The prevalence of schistosomiasis among school-age children $(76.8 \%)$ as evidenced by $S$. haematobium ova in urine, indicated that Abuselala exceeds this Mass Drug Administration (MDA) threshold and qualifies for annual MDA. In Alsafia the prevalence is $44.7 \%$ among school age children and thus merits MDA every two years (Table 3 ).

Table 3 Pattern of prevalence rates of S. haematobium among different age groups South Darfur, March 2010

\begin{tabular}{lll}
\hline Age group & Negative & Positive \\
\hline $0-4$ & $11(73.3 \%)$ & $4(26.7 \%)$ \\
$5-9$ & $171(51.8 \%)$ & $159(48.2 \%)$ \\
$10-14$ & $80(27.0 \%)$ & $216(73.0 \%)$ \\
$15-19$ & $20(30.8 \%)$ & $45(69.2 \%)$ \\
$20-24$ & $21(67.7 \%)$ & $10(32.3 \%)$ \\
$25-29$ & $22(64.7 \%)$ & $12(35.3 \%)$ \\
$30-34$ & $10(76.9 \%)$ & $3(23.1 \%)$ \\
$35-39$ & $7(58.3 \%)$ & $5(41.7 \%)$ \\
$>=40$ & $15(100.0 \%)$ & $0(0.0 \%)$ \\
\hline
\end{tabular}

Tailored health education for the community is required for the control and prevention of urinary schistosomiasis and its attendant illnesses in the study areas $[1,19,20]$. Inclusion of schistosomiasis in the primary school curriculum would help to address children in the school. Finally, MDA should not be a substitute for implementation through regular health services. Strengthening the health system and building the capacity of health workers in diagnosis and treatment of schistosomiasis remains the only option to address those ineligible for MDA.

\section{Interventions}

The result of the survey was presented in the weekly health coordination meetings with the resource required to conduct the intervention. Based on the request, multiple agencies committed resources for the MDA. Before the mass treatment, meetings were organized with community leaders and health education was provided through community health workers. The dosage of praziquantel was $40 \mathrm{mg} / \mathrm{kg}$ body weight. Praziquantel tablets were procured through the UNICEF Supply Division in Copenhagen, Denmark and shipped to Sudan. Individuals at least 5 years old were eligible for treatment. Exclusion criteria included reported hypertension measured on site, self-reported history of asthma, allergy, pregnancy, lactation, recent delivery, possible pregnancy, seizure disorder, past surgery or currently febrile state. The provision of praziquantel adhered to the Ministry of Health guidelines. Body weight was recorded and consumption of doses of praziquantel was directly observed by drug distributors. A total of 13,469 individuals received a dose of praziquantel.

\section{Acknowledgements}

We acknowledge the support received from the project community and State Ministry of Health and locality Health offices.

Funding

The work presented in this publication was made possible by the generous support of the American People through United States Agency for 
International Development Office of United States Foreign Disaster Assistance USAID/OFDA. USAID/OFDA funding was provided to American Refugee Committee International for health, WASH and Agriculture service delivery. The State Ministry of Health provided material support and coordinated the survey and MDA. UNICEF supplied Praziquantel for MDA. WHO provided technical support through training of drug distributors. The funders had no role in study design, data collection and analysis, or preparation of the manuscript.

Disclaimer: The views, the findings and conclusions represented in this article are those of the authors and do not necessarily represent the official views of their institutions where they are affiliated.

\section{Author details}

'American Refugee Committee International, Nyala, South Darfur, Sudan. ${ }^{2}$ State Ministry of health, Nyala, South Darfur state, Sudan. ${ }^{3}$ World Health Organization WHO South Darfur Sub Office Nyala, Sudan. ${ }^{4}$ UNICEF Nyala Office, South Darfur, Sudan. ${ }^{5}$ State Ministry of Health, Department of Epidemiology, Nyala, South Darfur, Sudan. ${ }^{6}$ State Ministry of Health; Malaria, Schistosomiasis and Leishmaniasis control program, Nyala, South Darfur, Sudan.

\section{Authors' contributions}

$\mathrm{KD}, \mathrm{GAA}, \mathrm{KB}, \mathrm{AA}$, AM designed the study. SH, FH, AEM, MDO assisted in data collection and analysis. TJ, EAM involved in designing the study and data collection. $K D, A E$, EK analyzed the data and wrote the first draft of the manuscript. All authors contributed to the manuscript and approved its final version.

\section{Competing interests}

The authors declare that they have no competing interests.

Received: 20 December 2010 Accepted: 7 February 2011 Published: 7 February 2011

\section{References}

1. Gryseels B, Polman K, Clerinx J, Kestens L: Human schistosomiasis. Lancet 2006, 368:1106-1118

2. World Health Organization: Preventive chemotherapy in human helminthiasis: coordinated use of anthelminthic drugs in control interventions: a manual for health professionals and programme managers. World Health Organization Geneva, Switzerland; 2006.

3. World Health Organization: Prevention and Control of Schistosomiasis and Soil-Transmitted Helminthiasis. Report of a WHO Expert Committee. WHO Technical Report Series No. 912 WHO, Geneva; 2002.

4. Van der Werf MJ, De vlas SJ, Brooker S, Looman CWN, Nagelkerke NJD, Habbema JDF, Engels D: Quantification of clinical morbidity associated with schistosome infection in sub-Saharan Africa. Acta Trop 2003, 86:125-139.

5. Lopez AD, Mathers CD, Ezzati M, Jamison DT, Murray CJL: Global burden of disease and risk factors. Oxford University Press/The World Bank, New YorkWashington; 2006.

6. King CH, Dickman K, Tisch DJ: Regauging the cost of chronic helminthic infection: meta-analysis of disability-related outcomes in endemic schistosomiasis. Lancet 2005, 365:1561-1569.

7. Beyrer C, Villar JC, Suwanvanichkij V, Singh S, Baral SD, et al: Neglected diseases, civil conflicts, and the right to health. Lancet 2007, 370:619-627.

8. State Ministry of Health: Annual report for the fiscal year 2009. State Ministry of Health, Nyala, Darfur; 2009

9. Montresor A, Crompton DWT, Hall A, Bundy DAP, Savioli L: Guidelines for the evaluation of soil-transmitted helminthiasis and schistosomiasis at community level. World Health Organization, Geneva; 1998.

10. World Health Organization: Sedimentation Method. IN Basic Laboratory Methods in Medical Parasitology. World Health Organization Geneva; 1991, 33-35.

11. World Health Organization Sudan: Darfur Weekly Report $18-24$ December 2005. [http://www.emro.who.int/sudan/media/pdf/Darfur\% 20weekly\%20report\%2018\%20-\%2024\%20December\%202005.pdf], Accessed on 5 May 2010.

12. Ahmed AA, Afifi AA, Adam I: High prevalence of Schistosoma haematobium infection in Gereida Camp, in southern Darfur, Sudan. Ann Trop Med Parasitol 2009, 103:741-3.
13. Doumenge JP, Mott KE, Cheung C, Villenave D, Chapuis O, et al: Atlas of the global distribution of schistosomiasis. Geneva: World Health Organization; 1987.

14. Abdel-Malek E: Distribution of the intermediate hosts of bilharziasis in relation to hydrography; with special reference to the Nile Basin and the Sudan. Bull World Health Organ 1958 1958, 18:691-734.

15. World Health Organization: Report, Inter-country Meeting on strategies to eliminate schistosomiasis from the eastern Mediterranean region Muscat, Oman 6-8 November 2007. [http://www.who.int/entity/ schistosomiasis/resources/EMRO_report_Schistosomiasis.pdf], Accessed on 5 May 2010

16. The Free Libraray: Schistosoma hematobium and S. mansoni among children, Southern Sudan. Diseases. [http://www.thefreelibrary.com/ Schistosoma+hematobium+and+S.+mansoni+among+children,+Southern +Sudan-a0169821869], Accessed on 5 May 2010.

17. Kabatereine NB, Brooker S, Tukahebwa EM, Kazibwe F, Onapa AW: Epidemiology and geography of Schistosoma mansoni in Uganda: implications for planning control. Trop Med Int Health 2004, 9:372-380.

18. Sturrock HJW, Picon D, Sabasio A, Oguttu D, Robinson E, et al: Integrated Mapping of Neglected Tropical Diseases: Epidemiological Findings and Control Implications for Northern Bahr-el-Ghazal State, Southern Sudan. PLoS Negl Trop Dis 2009, 3:e537.

19. Sow S, De Vlas SJ, Mbaye A, Polman K, Gryseels B: Low awareness of intestinal schistosomiasis in northern Senegal after 7 years of health education as part of intense control and research activities. Trop Med Int Health 2003, 8:744-749.

20. Engels D, Ndoricimpa J, Gryseels B: Schistosomiasis mansoni in Burundi: progress in its control since 1985. Bull World Health Organ 1993, 71:207-214.

doi:10.1186/1756-3305-4-14

Cite this article as: Deribe et al: High prevalence of urinary schistosomiasis in two communities in South Darfur: implication for interventions. Parasites \& Vectors 2011 4:14.

\section{Submit your next manuscript to BioMed Central and take full advantage of:}

- Convenient online submission

- Thorough peer review

- No space constraints or color figure charges

- Immediate publication on acceptance

- Inclusion in PubMed, CAS, Scopus and Google Scholar

- Research which is freely available for redistribution 